ARTICLE

Received 29 Aug 2014 | Accepted 6 Dec 2014 | Published 12 Jan $2015 \quad$ DOl: 10.1038/ncomms7048

\title{
The Hippo pathway effector YAP is a critical regulator of skeletal muscle fibre size
}

\author{
K.I. Watt ${ }^{1,2}$, B.J. Turner ${ }^{3}$, A. Hagg ${ }^{1}$, X. Zhang ${ }^{2,4,5}$, J.R. Davey ${ }^{1}$, H. Qian' ${ }^{1}$, C. Beyer ${ }^{1}$, C.E. Winbanks ${ }^{1}$, K.F. Harvey ${ }^{2,4,5}$ \\ \& P. Gregorevic $1,6,7,8$
}

The Yes-associated protein (YAP) is a core effector of the Hippo pathway, which regulates proliferation and apoptosis in organ development. YAP function has been extensively characterized in epithelial cells and tissues, but its function in adult skeletal muscle remains poorly defined. Here we show that YAP positively regulates basal skeletal muscle mass and protein synthesis. Mechanistically, we show that YAP regulates muscle mass via interaction with TEAD transcription factors. Furthermore, YAP abundance and activity in muscles is increased following injury or degeneration of motor nerves, as a process to mitigate neurogenic muscle atrophy. Our findings highlight an essential role for YAP as a positive regulator of skeletal muscle size. Further investigation of interventions that promote YAP activity in skeletal muscle might aid the development of therapeutics to combat muscle wasting and neuromuscular disorders.

\footnotetext{
${ }^{1}$ Baker IDI Heart and Diabetes Institute, Melbourne, Victoria 3004, Australia. ${ }^{2}$ Peter MacCallum Cancer Centre, East Melbourne, Melbourne, Victoria 3002, Australia. ${ }^{3}$ The Florey Institute of Neuroscience and Medical Health, The University of Melbourne, Melbourne, Victoria 3010, Australia. ${ }^{4}$ Sir Peter MacCallum Department of Oncology, The University of Melbourne, Melbourne, Victoria 3010, Australia. ${ }^{5}$ Department of Pathology, The University of Melbourne, Melbourne, Victoria 3010, Australia. ${ }^{6}$ Department of Biochemistry and Molecular Biology, Monash University, Melbourne, Victoria 3800 , Australia. ${ }^{7}$ Department of Physiology, The University of Melbourne, Melbourne, Victoria 3010, Australia. ${ }^{8}$ Department of Neurology, The University of Washington School of Medicine, Seattle, Washington 98195, USA. Correspondence and requests for materials should be addressed to P.G. (email: paul.gregorevic@bakeridi.edu.au).
} 
T he Hippo pathway is a critical regulator of cell division and apoptosis that governs organ size in multiple species ${ }^{1,2}$. Through diverse inputs that include apicobasal cell polarity, cell-cell adhesion, the tensile state of the actin cytoskeleton and G-protein-coupled receptor signalling ${ }^{1-6}$, the Hippo pathway suppresses the activity of the transcriptional coactivators Yes-associated protein (YAP) and WW domain containing protein 1 (TAZ/WWTR1) by post-translational regulation and/or nuclear exclusion ${ }^{1,2}$. In conditions of low Hippo signalling, YAP and TAZ are active and localize in the nucleus, where they associate with the transcriptional enhancer factor domain 1-4 (TEAD 1-4) transcription factors and derepress the expression of genes that control cell proliferation and terminal differentiation ${ }^{7}$. Despite a broad understanding of the function of the Hippo pathway in mitotically active tissues including the liver, skin, intestine and the developing heart ${ }^{8-17}$, the role of this pathway in adult, post-mitotic skeletal muscle fibres is poorly understood.

Circumstantial evidence supports a potential role for YAP as a positive regulator of cell size in striated musculature. In cardiomyoctytes, endogenous LATS2 (which promotes the inactivation of YAP) can negatively regulate growth ${ }^{18}$. These findings were recently substantiated by observations that increased YAP expression can promote cardiomyocyte growth ${ }^{19}$. In skeletal muscle, the overexpression of the mammalian homologue of the Hippo kinase (MST1) - which inactivates YAP-leads to atrophy of fast-twitch muscle fibres via FoxO-dependent transcription of the ubiquitin ligase Atrogin-1 (Fbxo32), and activation of autophagy ${ }^{20}$. These observations support the hypothesis that YAP may contribute to controlling the growth and remodelling of adult mammalian skeletal musculature in instances of physiological adaptation and disease.

Given the abundant expression of Hippo pathway elements in mature skeletal muscle ${ }^{20,21}$, and evidence supporting a role for the Hippo pathway as a regulator of cardiomyocyte growth ${ }^{18}$, we sought to test the hypothesis that YAP performs an essential role in the regulation of skeletal muscle fibre size. Using gain- and loss-of-function approaches, our findings demonstrate that YAP, the core effector of the Hippo pathway, is a mediator of size in adult skeletal muscle fibres, and an effector of processes that promote protein synthesis. In addition, we have established that the abundance of YAP in muscles is increased following disruption of the interaction between motor nerves and muscle fibres, and provide the first evidence of a functional role for YAP and TEAD in the preservation of muscle mass in conditions of atrophy associated with disruption of the NMJ.

\section{Results}

YAP levels decline during maturation of skeletal myofibres. To define the expression profile of YAP during the development and maturation of mammalian skeletal muscle fibres, we first examined the abundance of YAP throughout the fetal and postnatal maturation of limb muscles in C57BL/6 mice. We found that YAP was abundant in the tibialis anterior (TA) muscles of fetal (E17) mice, but declined during postnatal maturation (Fig. 1a, Supplementary Fig. 1a). Phosphorylation of YAP at serine 112 $\left(\mathrm{YAP}^{\mathrm{S} 12}\right.$ ) by the Hippo pathway kinases LATS1 and LATS2 promotes nuclear exclusion of YAP and represses its activity ${ }^{1,2}$. Levels of YAP ${ }^{\mathrm{S} 112}$ also declined between E17 and adult stages, but no difference in the ratio of $\mathrm{YAP}^{\mathrm{S} 112} / \mathrm{YAP}$ was observed, suggesting that YAP abundance diminished in concert with reduced Hippo pathway activity during this time (Fig. 1a, Supplementary Fig. 1b,c). Reduced YAP protein abundance was correlated with diminishing Yap mRNA expression, indicating that transcriptional regulation contributes to the determination of
YAP abundance in maturing muscles (Fig. 1b). To examine the localization of YAP, we performed immunofluorescent labelling of the TA muscles of 8-week-old C57BL/6 mice using antibodies against YAP and laminin (to identify the muscle fibre membrane), in conjunction with $4^{\prime}, 6$-diamidino-2phenylindole (to identify total nuclei) and fluorophoreconjugated bungarotoxin (to mark the neuromuscular junction). YAP labelling was clearly detectable in some nuclei localized within the laminin boundary of muscle fibres (Fig. 1c-l). YAP protein was also detected at the muscle fibre membrane. YAP was more diffuse at the membrane than in nuclei, although we observed an accumulation of YAP near the NMJ, where sarcolemmal density is increased. YAP was also detected within the nucleus, consistent with its localization of YAP in myogenic cell lines ${ }^{21}$.

YAP is required to maintain basal skeletal myofibre size. Having established that YAP levels in skeletal muscle change over the time course of postnatal muscle maturation where significant cell growth occurs, we sought to determine the functional requirement for YAP in the regulation of skeletal muscle fibre size. To test the effect of reduced YAP abundance on muscle attributes, we generated a recombinant adeno-associated viral vector (rAAV6) carrying an expression cassette that encodes for a short-hairpin RNA (shRNA) targeting mouse YAP (rAAV6:YAP shRNA), for administration into mouse limb muscles in vivo. This shRNA sequence has been used previously to target endogenous YAP in vitro ${ }^{22,23}$. As a control, contralateral muscles were injected with a rAAV6 vector that encoded a shRNA sequence targeting LacZ (rAAV6:LacZ shRNA). Mouse TA muscles examined 14 days after administration of rAAV6:YAP shRNA demonstrated a $72 \pm 7 \%$ (mean \pm s.e.m., $n=5$ ) reduction in endogenous YAP protein content, compared with contralateral muscles receiving rAAV6:LacZ shRNA (Fig. 2a). The observed reduction of YAP protein in muscles receiving rAAV6:YAP shRNA was associated with a significant decrease in muscle mass and myofibre cross-sectional area (CSA), with no evidence of fibre degeneration (Fig. 2b-d). To confirm the efficacy of YAP knockdown, we examined the expression of known YAP/TEAD target genes. In TA muscles administered with rAAV6:YAP shRNA, the expression of the established YAP target genes Ankrd1 and Ctgf was reduced $48 \pm 13 \%$ and $48 \pm 12 \%$ (mean \pm s.e.m., $n=5$ ), respectively (Fig. 2e,f). These findings show, for the first time, that YAP is a mediator of basal skeletal myofibre mass in vivo, and support the hypothesis that YAP positively regulates cell size in adult skeletal myofibres.

Maintenance of adult skeletal muscle mass is achieved by balancing the rates of protein synthesis and degradation ${ }^{24}$. We examined if muscle atrophy associated with YAP knockdown was a function of reduced protein synthesis, by using the SuNSET technique ${ }^{25}$, which measures acute incorporation of Puromycin. Muscles examined 14 days after injection of rAAV6:YAP shRNA demonstrated significantly reduced rates of protein synthesis compared with muscles receiving rAAV6:LacZ shRNA (Fig. 2g,h). No changes were observed in the phosphorylation of mammalian target of rapamycin (mTOR, a key mediator of protein synthesis and skeletal muscle growth ${ }^{24}$ ), or the mTOR substrates 4E-BP1 and p70S6K, between YAP shRNA and contralateral LacZ-shRNA-treated muscles, thereby demonstrating that the reduction in protein synthesis as a result of YAP shRNA administration was not associated with suppressed mTOR activity (Supplementary Fig. 2a-d). In skeletal muscle, protein degradation is predominately controlled by the ubiquitinproteasome system (UPS). To determine if muscle atrophy associated with YAP knockdown is a function of increased 
a

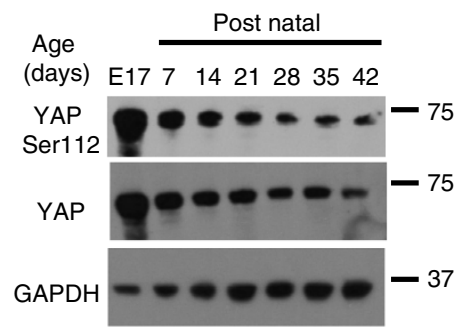

b

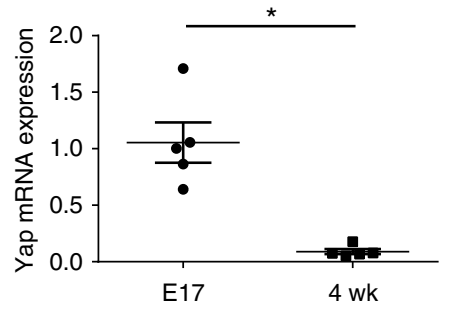

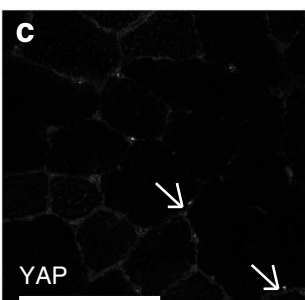
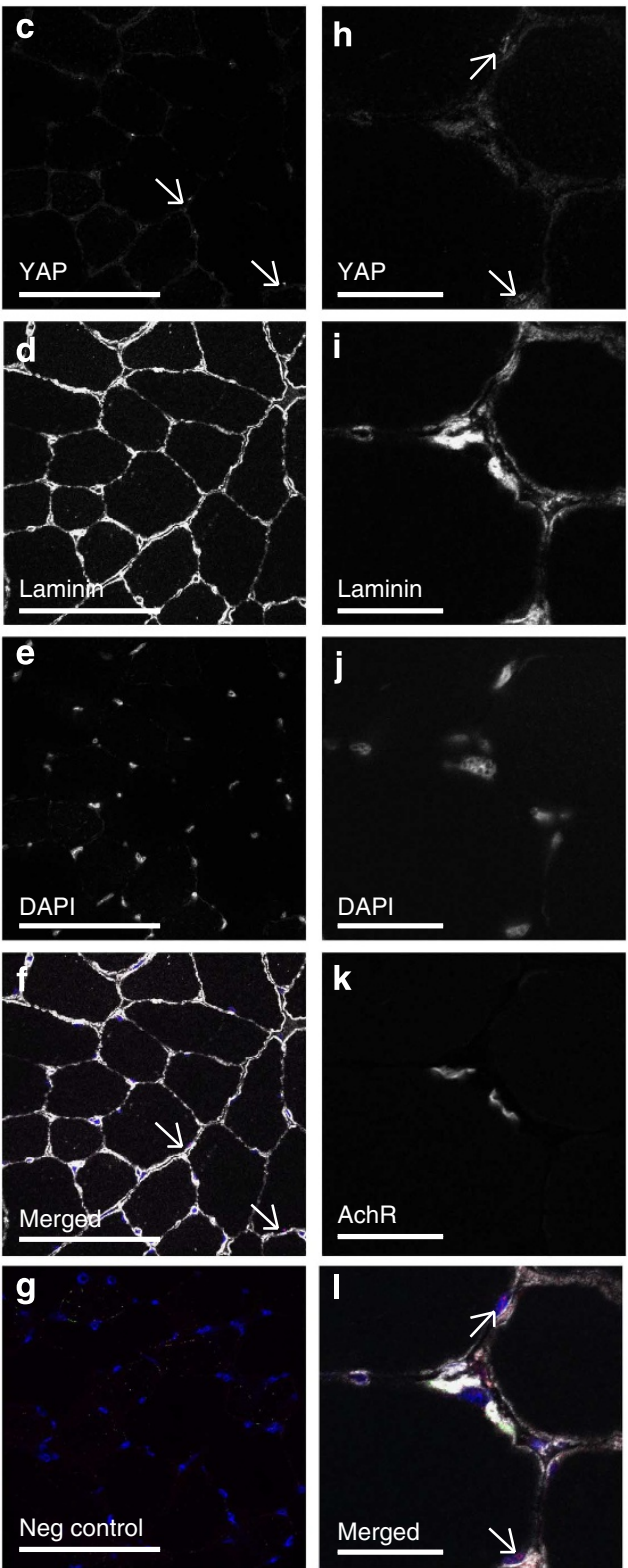

Figure 1 | YAP abundance decreases with postnatal maturation of skeletal muscle fibres. (a) Representative western blots showing YAPS112, YAP and GAPDH levels from embryonic day 17 (E17) throughout postnatal maturation until 42 days of age. $n=3$. (b) Yap mRNA expression between E17 and 4 weeks of age (mean \pm s.e.m., $n=5, P=0.0007$; unpaired $t$-test). (c) Representative image of YAP localization in the TA muscle of an 8-week-old C57BL/6 mouse, (d) Laminin labelling marking the muscle fibre membrane, (e) 4',6-diamidino-2-phenylindole (DAPI)-labelled nuclei, (f) Merged image of c-e. YAP is shown in red, DAPI in blue and laminin in grey. Arrows indicate YAP-positive muscle nuclei, ( $\mathbf{g}$ ) negative control of tissue section labelled with secondary antibody and DAPI only. (h-I) higher magnification images of YAP localization in muscle nuclei and diffuse membrane localization, accumulating at the membrane near the acetylcholine receptors (AchR) as labelled with $\alpha$-bungarotoxin conjugated to AlexaFluor594 and shown in green in I. Scale bar (c,g), $100 \mu \mathrm{m}$ and (h-I), $25 \mu \mathrm{m} . n=3$.

protein degradation, we measured the expression of the three key E3 ubiquitin ligases that mediate UPS activity in muscle (Atrogin-1, Murf1 and Musa1 (refs 24,26-28). No differences were observed in the expression of these genes in muscles treated with rAAV6:YAP shRNA or rAAV6:LacZ shRNA (Fig. 2i-k). These findings demonstrate that the YAP-mediated regulation of postnatal muscle mass is achieved by processes that influence protein synthesis, as opposed to UPS-mediated protein degradation.

YAP promotes hypertrophy via TEAD transcription factors. Having established that YAP knockdown yields a reduction in muscle mass, we sought to determine if the overexpression of YAP can promote skeletal muscle fibre hypertrophy. To increase YAP expression in the skeletal muscle fibres, we injected the TA muscles of 6-8-week-old C57BL/6 male mice with a rAAV vector carrying an expression cassette encoding for YAP2L (rAAV6:YAP), the predominant YAP isoform expressed in mouse skeletal muscle ${ }^{29}$. Muscles of the contralateral limb were injected with rAAV6:MCS (empty vector) as controls. Examination of muscles 4 weeks after the administration of rAAV6:YAP demonstrated that treatment led to the strong overexpression of YAP $(8.2 \pm 2$-fold, mean \pm s.e.m., $n=5)$, which was comparable to the differences observed in TA muscles between 1 and 6 weeks 

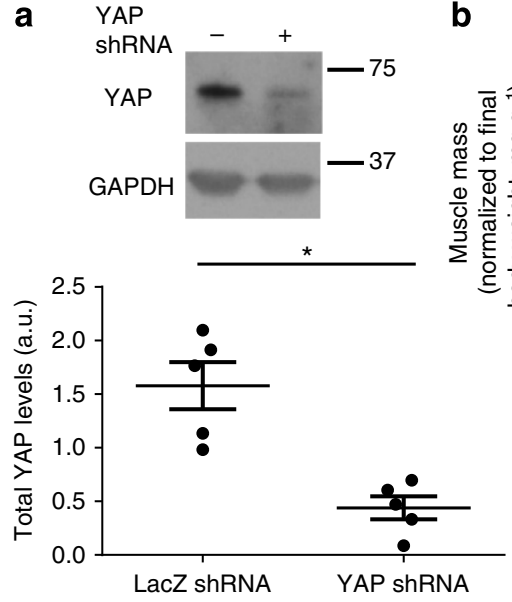

\section{d}

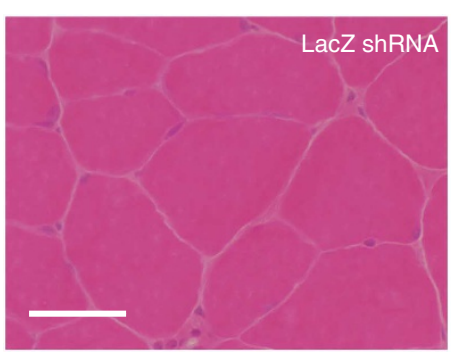

\section{f}
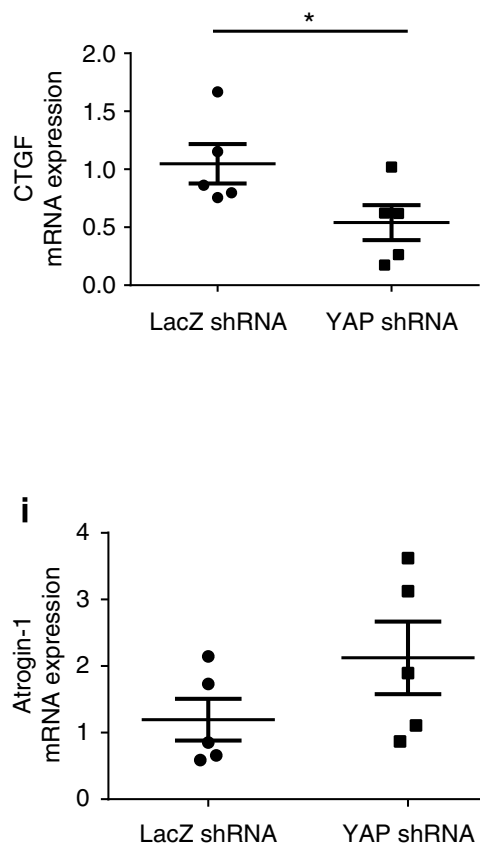

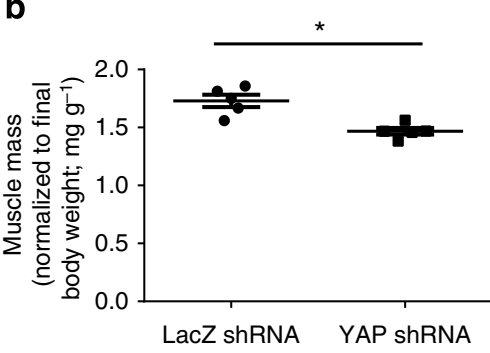

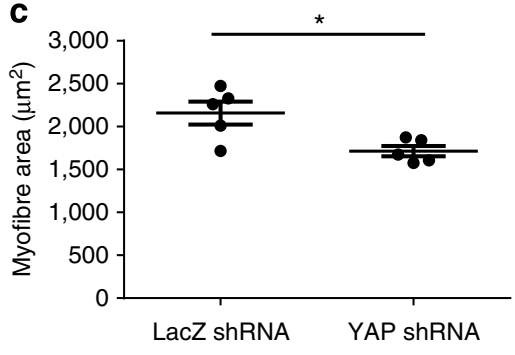

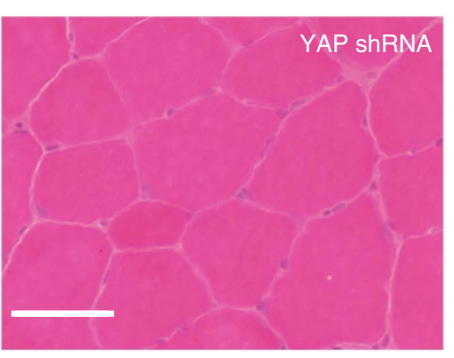

g
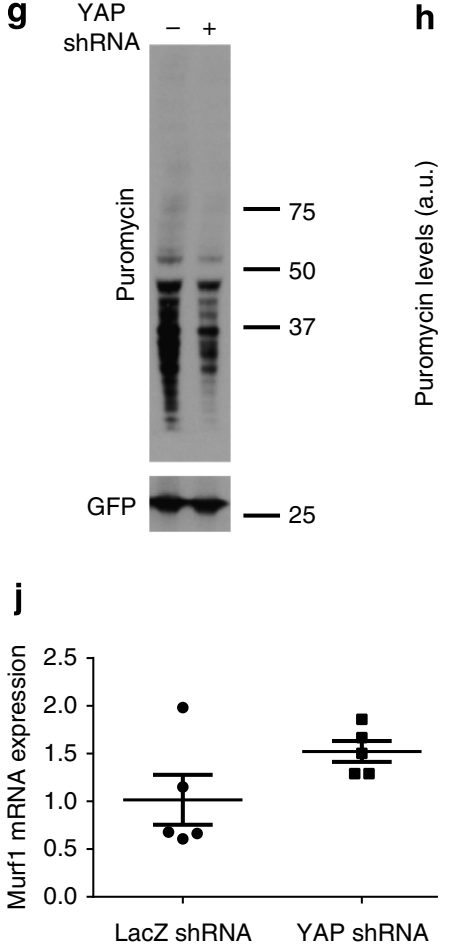

h
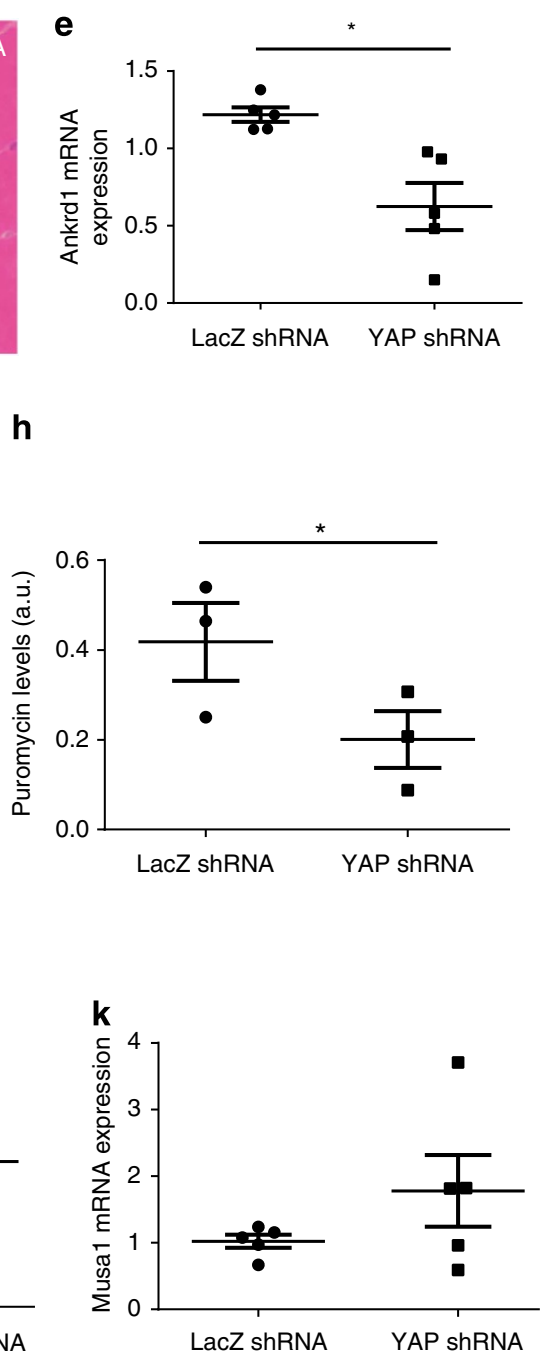

Figure 2 | YAP is required for the maintenance of basal skeletal muscle fibre size. (a) Representative western blot showing YAP and GAPDH levels in muscles treated with rAAV6:LacZ shRNA in the left TA muscle (LTA) or rAAV6:YAP shRNA in the right TA muscle (RTA) at a dose of $5 \times 10^{9}$ vector genomes for 2 weeks with quantification shown below. (mean \pm s.e.m., $n=5, P=0.03$, paired $t$-test, ${ }^{\star}$ indicates significance between groups). (b) Muscle mass of TA muscles treated with rAAV6:LacZ shRNA or rAAV6:YAP shRNA for 2 weeks. (mean \pm s.e.m., $n=5, P=0.003$, paired $t$-test, ${ }^{\star}$ indicates significance between groups). (c) CSA of rAAV6:LacZ shRNA- or rAAV6:YAP shRNA-treated myofibres after 2 weeks. (mean \pm s.e.m., $n=5, P=0.03$, paired $t$-test, ${ }^{*}$ indicates significance between groups). (d) Representative images of rAAV6:LacZ shRNA- or rAAV6:YAP shRNA-treated muscles after 2 weeks. Scale bar, $50 \mu \mathrm{m} . n=5$. (e) Ankrd1 and (f) Ctgf mRNA expression in rAAV6:LacZ shRNA- or rAAV6:YAP shRNA-treated muscles. (mean \pm s.e.m., $n=5, P=0.02$ and 0.01 respectively; paired $t$-test, ${ }^{*}$ indicates significance between groups). (g) Representative western blots and (h) quantification of Puromycin and GFP levels in rAAV6:LacZ shRNA or rAAV6:YAP shRNA treated muscles after 2 weeks. (mean \pm s.e.m., $n=3, P=0.04$, paired $t$-test, * indicates significance between groups). (i) Atrogin-1, (j) Murf1, (k) Musa1 mRNA expression in rAAV6:LacZ shRNA or rAAV6:YAP shRNA treated muscles after 2 weeks. (mean \pm s.e.m., $n=5, P=0.32,0.13$ and 0.26 respectively, paired $t$-test). 
of age (8.1 \pm 2 -fold, mean \pm s.e.m., $n=3$; Figs 1 a and $3 a)$. Importantly, muscles treated with rAAV6:YAP displayed a $13 \pm 4 \%$ increase in muscle mass, a $10 \pm 3 \%$ (mean \pm s.e.m., $n=4)$ increase in mean myofibre CSA and increased Ankrd1 mRNA expression (Fig. 3b-d). The hypertrophy observed in muscles administered rAAV6:YAP was associated with evidence of increased protein synthesis (Fig. 3e,f), but no effect on mTOR activity (Supplementary Fig. 2e,f) or UPS activity as measured by expression of Atrogin-1, Murf1 and Musal (Fig. 3j-1). In additional mice, administration of rAAV6:YAP to the soleus muscles elicited a comparable magnitude of hypertrophy, thereby demonstrating that the anabolic effects of increased YAP expression are observed in muscles of differing anatomy, fibre type composition and loading/recruitment pattern (Supplementary Fig. 3a-d). These results support the hypothesis that YAP positively regulates muscle mass, and that increasing YAP levels is sufficient to promote muscle hypertrophy in vivo.

In most settings examined to date, YAP associates with the TEAD transcription factors to control organ size $e^{2,30}$. We hypothesized that the interaction between YAP and TEAD is also required for the regulation of adult skeletal muscle mass. To test this potential mechanism, we co-injected mouse limb muscles with rAAV6:YAP and a rAAV vector expressing a dominantnegative mutant of TEAD2 (rAAV6:dnTEAD2). This mutant TEAD protein can bind to YAP, but lacks a DNA-binding domain. Whereas the administration of rAAV6:dnTEAD2 alone 4 weeks before examination did not significantly alter muscle mass or myofibre CSA (Supplementary Fig. 4a-e), the coadministration of rAAV6:dnTEAD2 with rAAV6:YAP blunted YAP induced muscle hypertrophy (Fig. 3a-c). No evidence of myofibre turnover or degeneration was observed in any of the muscles examined (Fig. 3g-i).

YAP can bind to each of the four TEAD transcription factors ${ }^{30}$. Since the expression of dnTEAD2 did not completely inhibit the hypertrophic effect of YAP overexpression in muscle, we sought an alternate approach to conclusively test the requirement for interactions between YAP and TEAD in the regulation of muscle size. Subsequently, we developed a rAAV vector expressing a mutant form of YAP2L that does not bind to any TEAD protein (rAAV6:YAP ${ }^{\mathrm{S} 79 \mathrm{~A}}$ ). Mutation of the Ser79 site within the $\mathrm{C}$ terminus of YAP has been shown to inhibit binding to all four TEAD proteins in vitro and in vivo ${ }^{11,30}$. Consistent with our hypothesis, muscles treated with rAAV6:YAPS79A 4 weeks prior displayed no difference in muscle mass and myofibre CSA compared with muscles receiving rAAV6:MCS (Supplementary Fig. 5a-e). Together these experiments demonstrate that YAP regulates the size of adult skeletal muscle fibres via interaction with TEAD transcription factors.

To determine the maximum effect of YAP overexpression on muscle mass and muscle fibre size, we treated the limb muscles of additional cohorts of mice with increasing quantities of rAAV6:YAP. Mice treated with up to $1 \times 10^{10}$ vector genomes displayed supraphysiological levels of YAP protein in rAAV6:YAP-treated muscles (12 \pm 0.6 -fold versus contralateral, mean \pm s.e.m., $n=5$ ) and evidence of notable hypertrophy as early as 10 days post injection (Supplementary Fig. 6a,b). However, after this time, we observed that the overexpression achieved with this dose induced a phenotype of muscle fibre degeneration (Supplementary Fig. 6c,d). A similar phenotype was reported using a transgenic mouse model engineered to express human YAP1 protein ${ }^{31}$. To identify if this phenotype is a result of general cellular toxicity from high protein expression, we assessed the effect of expressing mutant YAP proteins at this dose. Interestingly, while overexpressing a constitutively active YAP2L (rAAV6:YAP ${ }^{\mathrm{S} 112}$ ) and YAP2Llacking WW-regulatory binding domains (rAAV6:YAPWWmutant) recapitulated the degeneration associated with the supraphysiological expression of wild-type YAP2L (Supplementary Fig. 6e-h), muscles expressing equivalently high levels of YAP ${ }^{\text {S79A }}$ did not exhibit pathology (Supplementary Fig. 6i,j).

YAP levels are elevated in denervated skeletal muscles. Denervation of skeletal muscle fibres results in accumulation of the Hippo pathway kinase MST1 (ref. 20). Having observed that YAP accumulates in adult muscle fibres at the membrane near the NMJ, we hypothesized that YAP activity and localization might be altered in muscle fibres following disruption or loss of the nerve-muscle interaction. In mice where we performed surgical resection of the peroneal nerve, which innervates muscles in the anterior compartment of the hind limb, we found that YAP transcription, phosphorylation at Ser112 and total protein abundance increased dramatically 3,7 and 14 days post denervation (Fig. 4a,b, Supplementary Fig. 7a). This effect was rapid since increases in YAP abundance were detectable within $6 \mathrm{~h}$ after denervation ( $150 \pm 9 \%$, mean \pm s.e.m., $n=4$; Fig. 4 c). No difference was observed in the ratio of $\mathrm{YAP}^{\mathrm{S} 112}$ /YAP protein in muscles examined after denervation, indicating that accumulation of YAP occurred concurrent with increased activity of the upstream Hippo pathway (Fig. 4b, Supplementary Fig. 7b,c). Importantly, probing cryosections of denervated muscles using antibodies against YAP revealed that denervation resulted in increased YAP accumulation within muscle nuclei (Fig. 4d-m, Supplementary Fig. 7d,e). To determine whether increased abundance of nuclear YAP is a hallmark of muscles undergoing neurogenic atrophy in a disease setting, we examined hind limb muscles from SOD1 G93A mice, the established model of amyotrophic lateral sclerosis. Consistent with our observations of experimentally denervated muscles, we observed that YAP expression and abundance were also increased in the TA muscles of SOD 1 G93A mice, examined at presymptomatic (60 days) and early symptomatic (90 days) stages (Supplementary Fig. 8a-e), and the gastrocnemius muscles of SOD1 ${ }^{\text {G93A }}$ mice examined at 90 days (Supplementary Fig. $8 \mathrm{f}-\mathrm{i}$ ).

To assess if the accumulation of YAP protein in muscles observed during neurogenic atrophy was dependent on perturbed NMJ function or altered mechanical loading, we measured pYAP $\mathrm{S}^{\mathrm{N} 12}$ and YAP levels in additional cohorts of mice examined 3 and 7 days after performing tenotomy of the gastrocnemius muscles (contralateral muscles were sham operated as controls). Tenotomized muscles displayed evidence of decreased muscle mass (Supplementary Fig. 9a), but no difference was observed in pYAP $^{S 112}$, total YAP or the ratio of $\mathrm{YAP}^{\mathrm{S} 112} / \mathrm{YAP}$ in this setting (Supplementary Fig. 9b-e). These data support the conclusion that the observed changes in YAP phosphorylation and abundance in muscles undergoing neurogenic atrophy are a consequence of disruption of the nerve-muscle interaction.

Our findings from manipulating YAP in healthy muscle fibres show that YAP is a positive regulator of skeletal muscle mass. We hypothesized that YAP is induced in muscles undergoing neurogenic atrophy, to promote growth and preserve mass. To test this hypothesis in a clinically relevant model of neurogenic atrophy, we treated contralateral muscles of SOD1 ${ }^{\mathrm{G} 93 \mathrm{~A}}$ female mice at a presymptomatic stage of the disease (60 days) with rAAV6:YAP or rAAV6:MCS for 30 days. Muscles treated with rAAV6:YAP displayed no difference in muscle mass, myofibre CSA or histological profile compared with contralateral muscles receiving rAAV6:MCS (Supplementary Fig. 10a-e). The data indicate that elevating total YAP levels during early symptomatic stages in SOD1 ${ }^{\mathrm{G} 93 \mathrm{~A}}$ mice is not sufficient to protect muscles from progressive atrophy.

To assess if YAP knockdown exacerbated muscle atrophy in SOD1G93A mice, we treated contralateral muscles with 

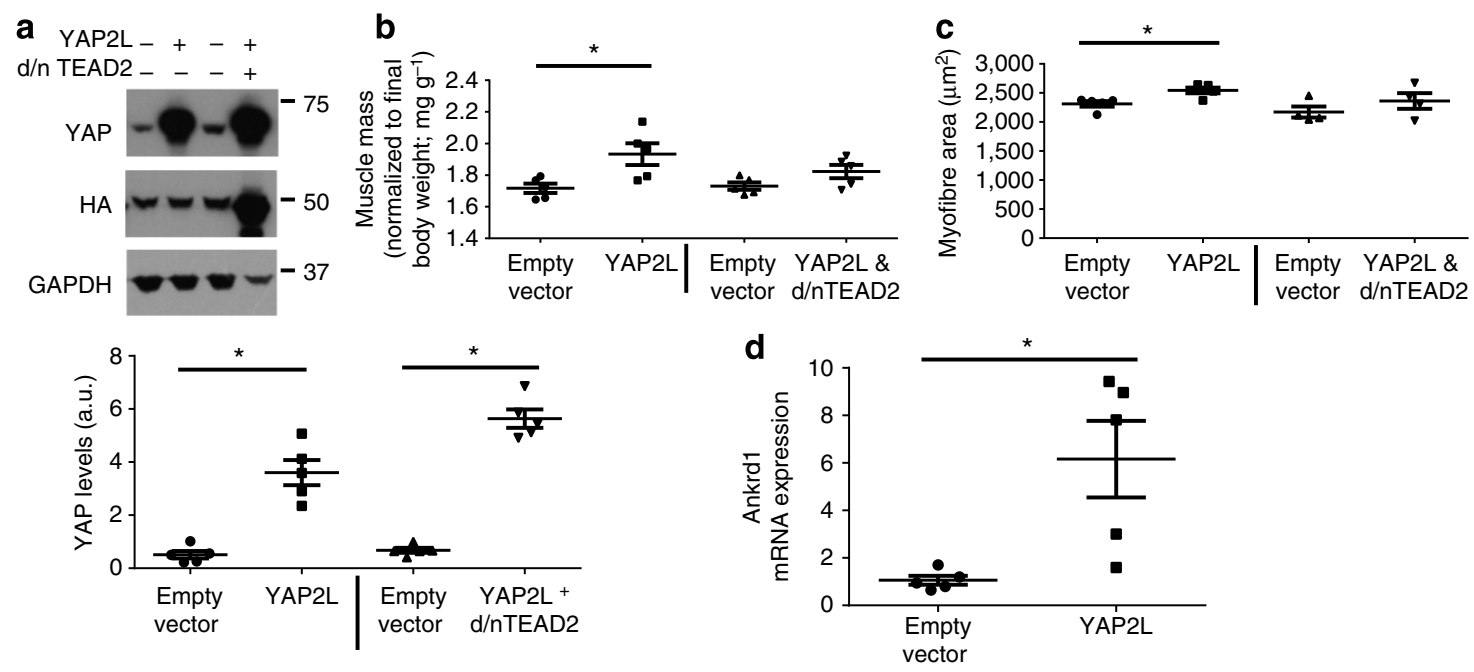

e

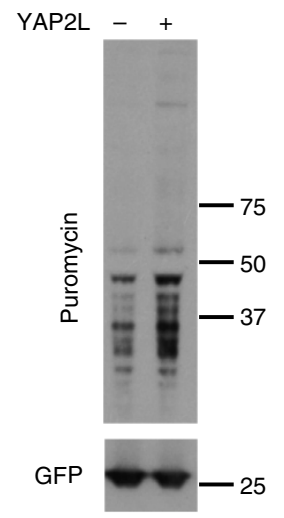

f

g

h
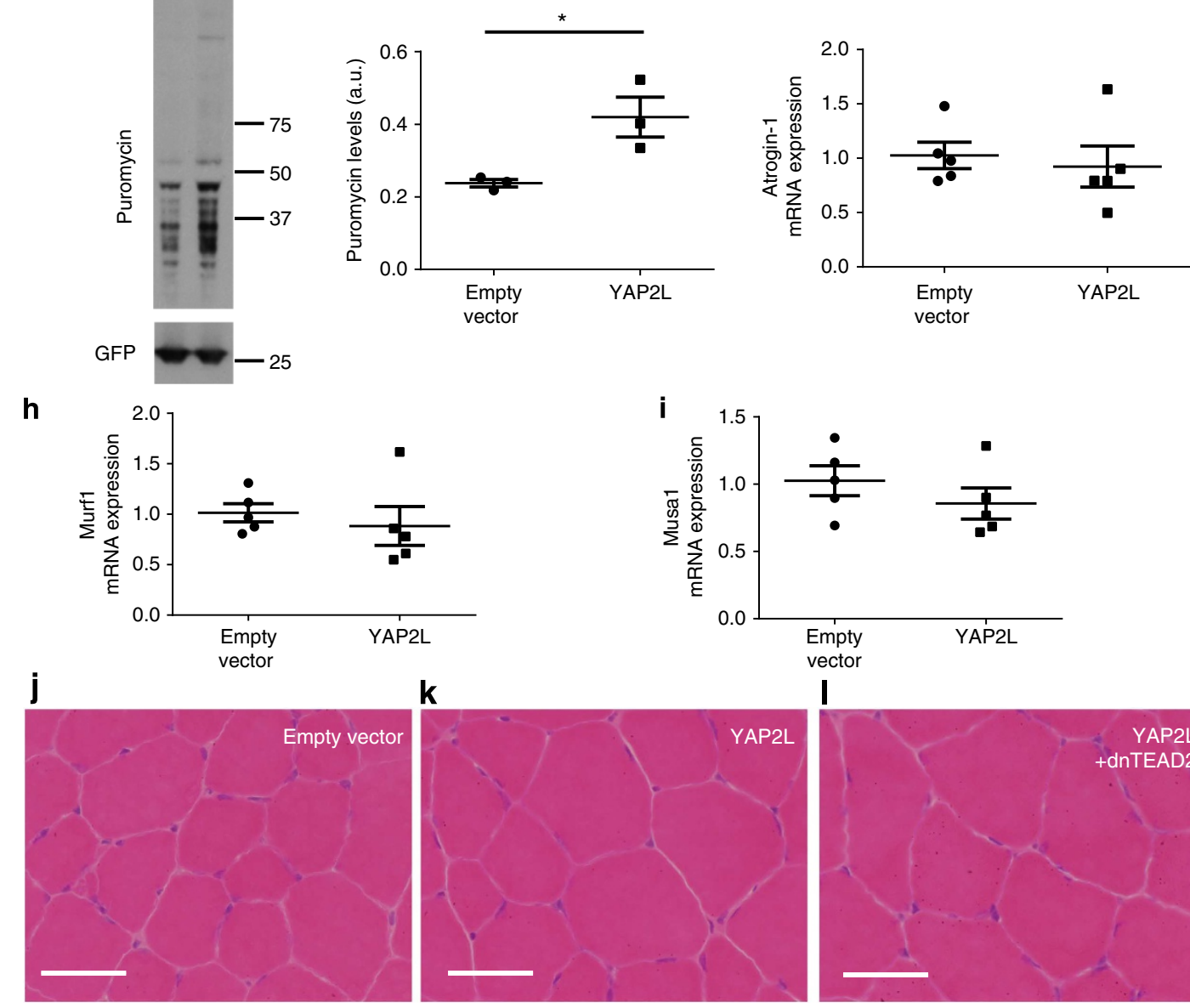

i

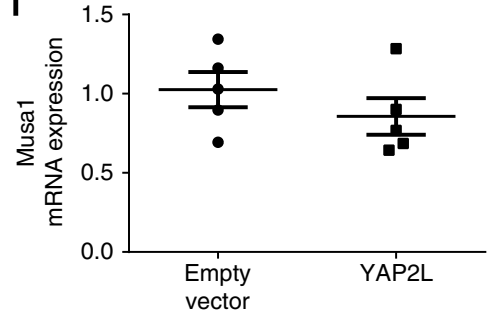

I

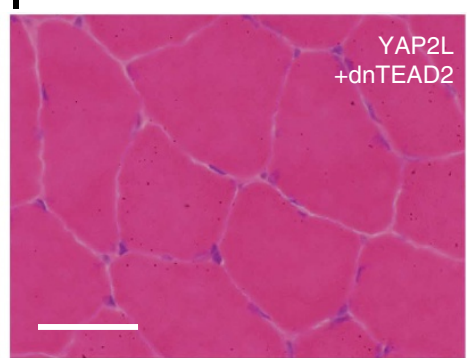

Figure 3 | YAP promotes skeletal muscle fibre hypertrophy via TEAD transcription factors. (a) Representative western blots showing YAP, HA-dnTEAD2 and GAPDH levels in muscles treated with either rAAV6:MCS in the LTA and rAAV6:YAP in the RTA or rAAV6:MCS in the LTA and rAAV6:YAP plus rAAV6:dnTEAD2 in the RTA with quantification shown below. (mean \pm s.e.m., $n=5, P=0.02$ and $<0.0001$ respectively; paired $t$-test, * indicates significance between groups). rAAV6:YAP and rAAV6:dnTEAD2 were both administered at a concentration of $1 \times 10^{9}$ vector genomes for 4 weeks. (b) Muscle mass of TA muscles of mice treated with rAAV6:MCS in the LTA and rAAV6:YAP in the RTA or rAAV6:MCS in the LTA and rAAV6:YAP plus rAAV6:dnTEAD2 in the RTA for 4 weeks. (mean \pm s.e.m., $n=5, P=0.02$ and 0.07 respectively; paired $t$-test, ${ }^{*}$ indicates significance between groups). (c) CSA of myofibres treated with rAAV6:MCS in the LTA and rAAV6:YAP in the RTA or rAAV6:MCS in the LTA and rAAV6:YAP plus rAAV6:dnTEAD2 in the RTA for 4 weeks. (mean \pm s.e.m., $n=4, P=0.03$ and 0.11 respectively; paired $t$-test, ${ }^{*}$ indicates significance between groups). (d) Ankrd1 mRNA expression in rAAV6:MCS- and rAAV6:YAP-treated muscles after 4 weeks (mean \pm s.e.m., $n=5, P=0.02$; paired $t$-test, * indicates significance between groups). (e) Puromycin and GFP levels with (f) quantification in rAAV6:MCS $(-)$ and rAAV6:YAP $(+)$ treated muscles after 4 weeks (mean \pm s.e.m., $n=3, P=0.04$; paired $t$-test, ${ }^{*}$ indicates significance between groups). (g) Atrogin-1, (h) Murf1, (i) Musa1 mRNA expression in rAAV6:MCS and rAAV6:YAP-treated muscles after 4 weeks (mean \pm s.e.m., $n=5, P=0.67,0.53$ and 0.16 respectively; paired $t$-test). (j-I) Representative images of rAAV6:MCS, rAAV6:YAP and rAAV6:YAP plus rAAV6:dnTEAD2-treated muscles after 4 weeks. Scale bar, $50 \mu \mathrm{m} . n=4$. 
a

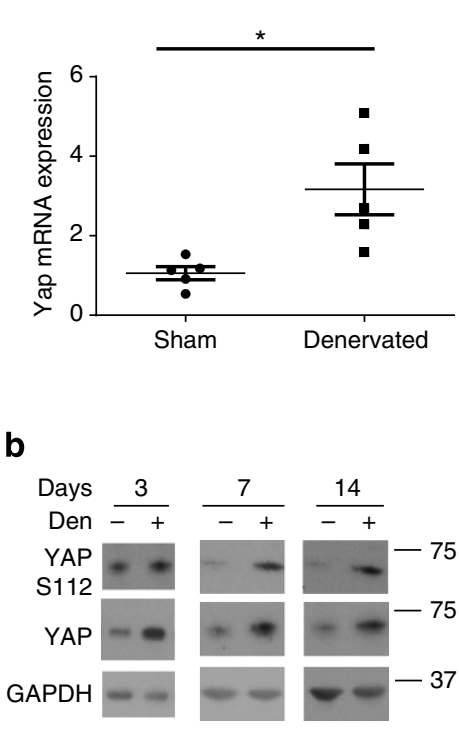

C

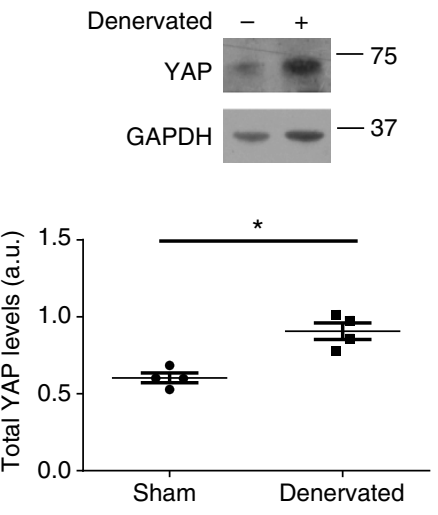

d
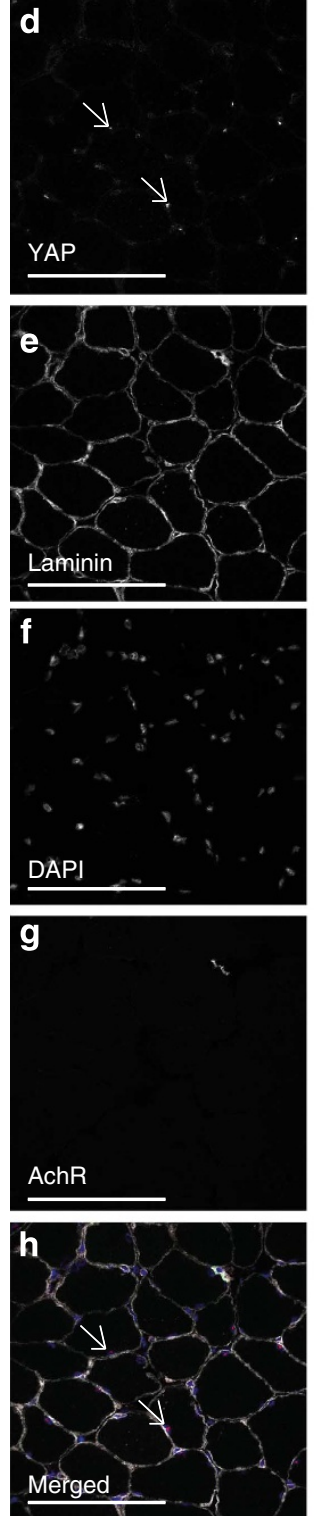
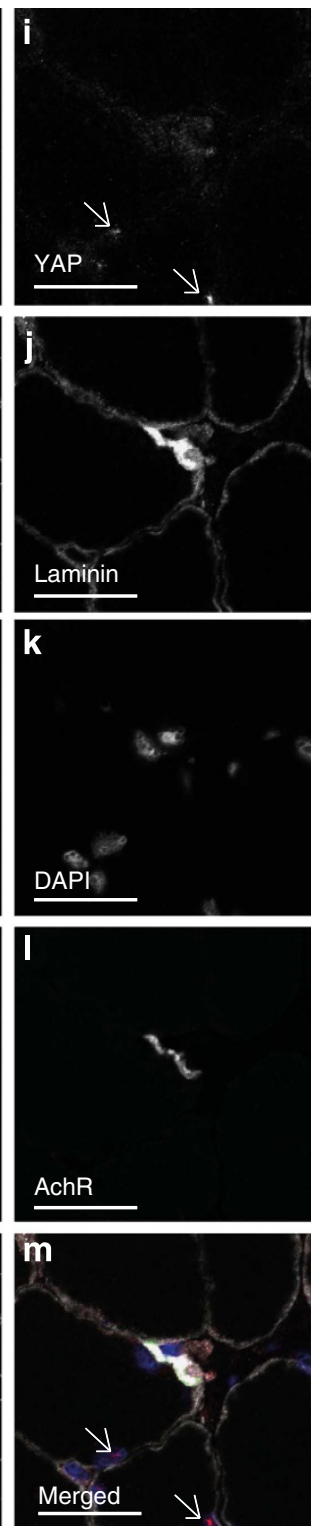

Figure 4 | YAP is elevated in skeletal muscle in response to denervation and neurodegeneration. (a) Yap mRNA expression in sham and denervated C57BL/ 6 adult male mouse TA muscles after 7 days (mean \pm s.e.m., $n=4, P=0.007$; paired $t$-test, ${ }^{\star}$ indicates significance between groups). (b) Representative western blots of YAPS112, YAP and GAPDH in sham $(-)$ and Den (denervated; + ) TA muscles after 3, 7 and 14 days as indicated. $n=4-5$. (c) YAP and GAPDH levels with quantification in sham $(-)$ and Den (denervated; + ) muscles $6 \mathrm{~h}$ after resection of the tibial branch of the peroneal nerve (mean \pm s.e.m., $n=4, P=0.02$; paired $t$-test, ${ }^{*}$ indicates significance between groups). (d) YAP, (e) Laminin, (f) 4',6-diamidino-2-phenylindole (DAPI) and (g) AchR localization of denervated TA muscles after 7 days. $n=3$. Scale bar on all images, $100 \mu \mathrm{m}$. (h) Merged image of e-g. YAP is shown in red, DAPI in blue, Laminin in grey and AchR in green. (i-m) Higher magnification images in 7-day denervated muscles. Scale bar on all images, $25 \mu \mathrm{m}$.

rAAV6:YAP shRNA or rAAV6:LacZ shRNA. The muscles of SOD1 ${ }^{\mathrm{G} 93 \mathrm{~A}}$ mice that were examined 30 days after receiving rAAV6:YAP shRNA contained $62 \pm 4 \%$ less YAP protein (mean \pm s.e.m., $n=5$; Supplementary Fig. 10f). While YAP knockdown elicited a reduction in muscle mass and myofibre CSA compared with muscles receiving rAAV6:LacZ shRNA (Supplementary Fig. $10 \mathrm{~g}-\mathrm{j}$ ), the magnitude of muscle atrophy was not greater in this setting than was observed in C57BL/6 mice treated with rAAV6:YAP shRNA (Fig. 2b,c).

YAP is upregulated to mitigate neurogenic muscle atrophy. SOD1 ${ }^{\mathrm{G} 93 \mathrm{~A}}$ mice model chronic, progressively severe neuromuscular disease, in which multiple mechanisms contribute to skeletal muscle atrophy ${ }^{32}$. To more precisely clarify the functional significance of elevated YAP levels in the setting of acute neurogenic muscle atrophy, we chose to study the response of muscles to experimental denervation. In contrast to the muscles of SOD1 ${ }^{\mathrm{G} 93 \mathrm{~A}}$ mice, the denervation of muscles in wild-type mice ensures that all myofibres within a muscle can be examined on a consistent time course and at specific times following loss of innervation.

To control for the effect of YAP shRNA on basal muscle mass, we administered either rAAV6:LacZ shRNA or rAAV6:YAP shRNA to the TA muscles of contralateral hind limbs 14 days before subsequently performing denervation in one limb (and a sham procedure in the contralateral leg). Innervated muscles examined 28 days after administration of rAAV6:YAP shRNA 
displayed comparable reductions in mass as observed in muscles analysed 14 days after rAAV6:YAP shRNA injection $(15 \pm 1 \%$ versus $15 \pm 2 \%$ respectively, mean \pm s.e.m., $n=3$; Supplementary Fig. 11a,b), validating this approach. Consistent with our earlier observations, YAP levels were markedly increased in muscles examined 7 days after denervation, and YAP abundance was markedly decreased in sham-operated muscles treated with rAAV6:YAP shRNA (Fig. 5a). Importantly, the administration of rAAV6:YAP shRNA significantly ablated the induction of YAP following denervation (Fig. 5a). Whereas muscles that were treated with rAAV6:LacZ shRNA and then examined 7 days after denervation exhibited a $13 \pm 3 \%$ reduction in mass (relative to the rAAV6:LacZ-shRNA-treated, sham-operated contralateral limb; Fig. 5b), the magnitude of atrophy caused by denervation of muscles previously administered rAAV6:YAP shRNA was more than doubled, relative to contralateral rAAV6:YAP shRNAtreated, sham-operated muscles $(-32 \pm 10 \%$, mean \pm s.e.m., $n=6$ except rAAV6:YAP shRNA where $n=5$; Fig. $5 \mathrm{~b}$ ). Histological examination confirmed that reductions in myofibre CSA as a consequence of denervation were also exacerbated in muscles where YAP knockdown had been achieved ( $-22 \pm 6 \%$ compared with rAAV6:LacZ-shRNA-treated denervated muscle, mean $\pm^{-}$ s.e.m., $n=6$ except rAAV6:YAP shRNA where $n=5$; Fig. $5 c-e$ ). The efficacy of YAP knockdown via prior rAAV6:YAP shRNA administration was confirmed by the marked suppression of ANKRD1 levels, compared with rAAV6:LacZ-shRNA-treated denervated muscles (Fig. 5f). These results demonstrate that the induction of YAP in skeletal muscle in response to loss of innervation limits neurogenic muscle atrophy.

Having established that YAP positively regulates protein synthesis in innervated muscles, we investigated whether the mitigating effects of YAP on neurogenic muscle atrophy related to rates of protein synthesis. Previous reports have shown that atrophy in denervated skeletal muscles leads to increased rates of protein synthesis due to stimulation of mTOR signalling by free amino acids generated during UPS-mediated protein breakdown ${ }^{32}$. Consistent with these findings and the enhanced atrophy, we observed in rAAV6:YAP shRNA-treated denervated muscles, we found that denervation of muscles previously administered rAAV6:LacZ shRNA displayed increased protein synthesis, and that the induction of protein synthesis was enhanced in denervated muscles previously treated with rAAV6:YAP shRNA (Fig. 5g,h). No difference was detected in the relative induction of the E3 ubiquitin ligases Atrogin-1, Murf1 and Musa1 in muscles following denervation, regardless of prior injection with rAAV6:YAP shRNA or rAAV6:LacZ shRNA (Fig. 5i-k). These data suggest that the enhanced atrophy and protein synthesis seen in denervated muscles receiving rAAV6:YAP shRNA is not due to increased UPS activity.
Inhibition of TEAD2 exacerbates neurogenic muscle atrophy. Having determined that the interaction with TEAD transcription factors is required for YAP to exert anabolic effects in healthy muscles, we investigated whether TEADs are also required to promote growth in the setting of neurogenic atrophy. Here mice received intramuscular injections of rAAV6:MCS and rAAV6:dnTEAD2 in the left and right TA muscles, respectively, $72 \mathrm{~h}$ before bilateral denervation. Examination of muscles 7 days after denervation demonstrated that neurogenic atrophy was exacerbated in muscles administered rAAV6:dnTEAD2, compared with muscles receiving rAAV6:MCS (Fig. 6a,b). Concomitant with this effect, myofibre CSA was reduced in three out of four mice treated with rAAV6:dnTEAD2 compared with contralateral denervated muscles receiving rAAV6:MCS (Fig. 6ce). Further, denervated muscles treated with rAAV6:dnTEAD2 displayed suppressed rates of protein synthesis compared with denervated muscles administered rAAV6:MCS (Fig. 6f,g). No difference was observed in Atrogin-1, Murf1 or Musa1 expression in denervated muscles previously administered either vector (Fig. $6 \mathrm{~h}-\mathrm{j}$ ). The data demonstrate that TEAD2 is required for the induction of protein synthesis in muscles in response to denervation and provide evidence that YAP and TEAD transcription factors positively regulate the mass of both healthy and denervated skeletal muscles.

\section{Discussion}

While YAP and the Hippo pathway predominately control organ size in epithelial tissues by altering the rates of cell proliferation and the activation of stem cells ${ }^{1,2,23}$, our findings provide the first evidence to show that the key Hippo pathway transcriptional regulatory protein, YAP, can also regulate the size of post-mitotic adult skeletal muscle fibres, by way of increasing protein synthesis. Our findings are consistent with those of previous studies that reported increased rates of protein synthesis in cardiomyocytes overexpressing a dominant-negative form of LATS2 (which normally represses YAP) ${ }^{18}$. The findings reported here demonstrate that YAP functions together with TEAD transcription factors in skeletal muscle and so extend our understanding of the importance of YAP and TEAD in the regulation of mammalian organ size in the postnatal environment.

A key finding of our study is the increased activity and nuclear accumulation of YAP in adult skeletal muscle fibres in response to disruption of the NMJ. An increase in YAP nuclear localization is consistent with that reported in $\mathrm{C} 2 \mathrm{C} 12$ myoblasts where active YAP promotes myoblast proliferation ${ }^{21}$. In addition to our demonstration that YAP participates as a mediator of basal muscle mass, our findings provide further support for a role for

\footnotetext{
Figure 5 | Endogenous YAP is upregulated to mitigate neurogenic muscle atrophy. (a) Representative western blots of YAP and GAPDH levels in sham or denervated muscles treated with rAAV6:LacZ shRNA or rAAV6:YAP shRNA with quantification below (mean \pm s.e.m., $n=6$ except $r A A V 6: Y A P$ shRNA denervated group where $n=5$, * indicates significant difference compared with sham rAAV6:LacZ muscle, \# indicates significant difference compared with rAAV6:LacZ shRNA denervated muscle, $P<0.05$; two-way analysis of variance (ANOVA) with Bonferroni post hoc test. (b) Muscle mass and (c) myofibre CSA in sham or denervated muscles treated with rAAV6:LacZ shRNA or rAAV6:YAP shRNA (mean \pm s.e.m., $n=6$ except $r A A V 6: Y A P$ shRNA denervated group where $n=5,{ }^{*}$ indicates significant difference compared with rAAV6:LacZ shRNA sham muscle, \# indicates significant difference compared with rAAV6:LacZ-shRNA-treated denervated muscle, \$ indicates significant difference compared with rAAV6:YAP shRNA-treated sham muscle, $P<0.05$; two-way ANOVA with Bonferroni post hoc test. (d,e) Representative images of muscles administered rAAV6:LacZ shRNA and rAAV6:YAP shRNA before denervation. Scale bar, $50 \mu \mathrm{m} . n=6$ except rAAV6:YAP shRNA denervated group where $n=5$. (f) Representative images of ANKRD1 and GAPDH levels in muscles receiving rAAV6:LacZ shRNA and rAAV6:YAP shRNA before denervation. $n=3$. (g) Puromycin and GFP levels with (h) quantification in rAAV6:LacZ shRNA and rAAV6:YAP shRNA-treated sham and denervated muscles (mean \pm s.e.m., $n=3$, * indicates significant difference compared with sham muscle, \# indicates significant difference compared with rAAV6:LacZ shRNA denervated muscle, $P<0.05$; two-way ANOVA with Bonferroni post hoc test. (i) Ankrd1, (j) Murf1 and (k) Musa1 mRNA expression in rAAV6:LacZ shRNA and rAAV6:YAP shRNA-treated sham and denervated muscles (mean \pm s.e.m., $n=6$ except rAAV6:YAP shRNA denervated group where $n=5$, * indicates significant difference compared to contralateral sham muscles, $P<0.05$ respectively; two-way ANOVA with Bonferroni post hoc test.
} 
the Hippo pathway in the control of muscle mass in neurogenic atrophy (Fig. 6 and ref. 20). While we found that the levels of phosphorylated $\mathrm{YAP}^{\mathrm{S} 112}$ and total YAP levels were elevated in denervated muscles, and in the muscles of $\mathrm{SOD} 1^{\mathrm{G} 93 \mathrm{~A}}$ mice (a change normally associated with increased activity of the Hippo pathway), we observed that YAP accumulated within the nuclei of denervated muscles (a process typically inhibited by Hippo signalling). Further, contrary to the overexpression of
MST1, we observed no change in the expression of the ubiquitin ligase Atrogin-1 in denervated muscles receiving rAAV6:YAP shRNA. Together these observations suggest that YAP activity could be regulated in denervated muscles by mechanisms aside from the core Hippo pathway kinases.

While changes in cell density and soluble factors such as GPCR ligands $1,6,30,33$ regulate YAP activity and nuclear localization via LATS1/2-dependent phosphorylation, modulation of the tensile a
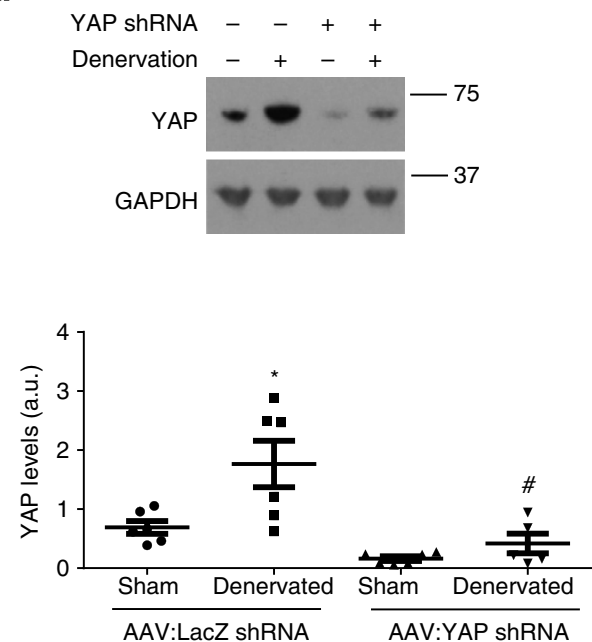

d

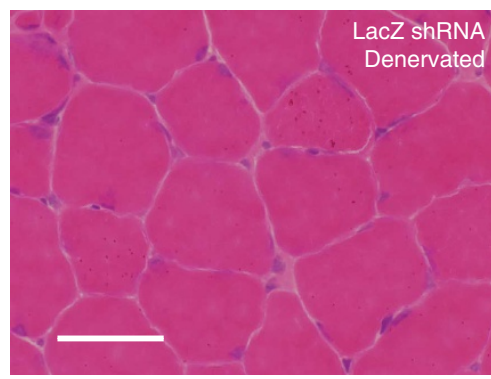

g

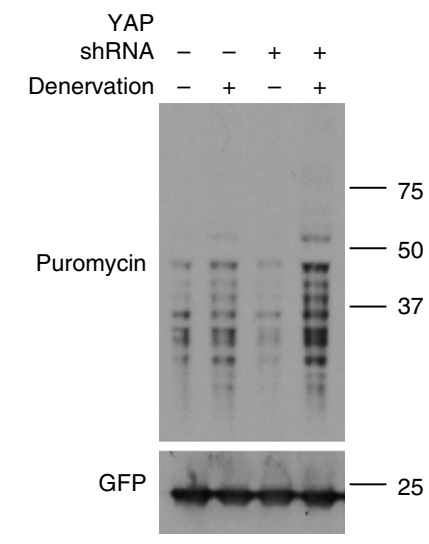

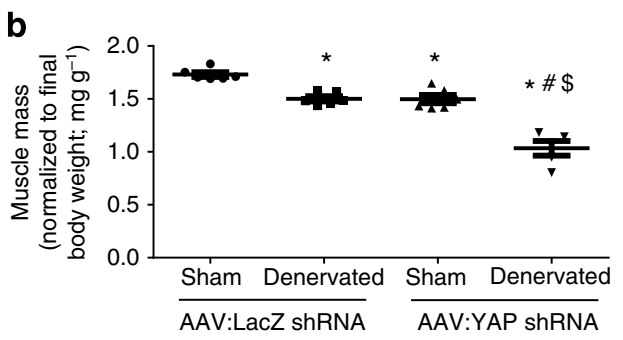

C

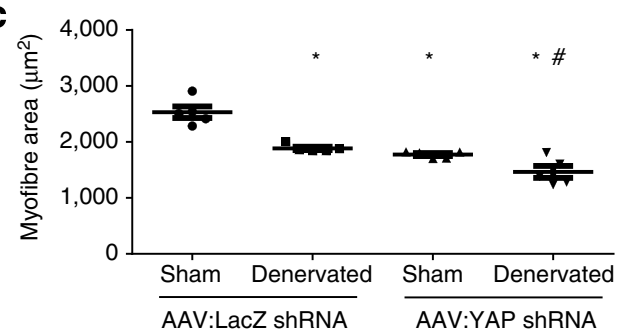

e

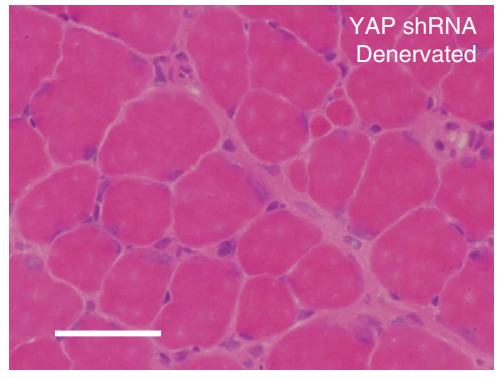

$f$

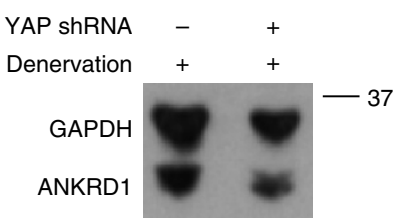

h
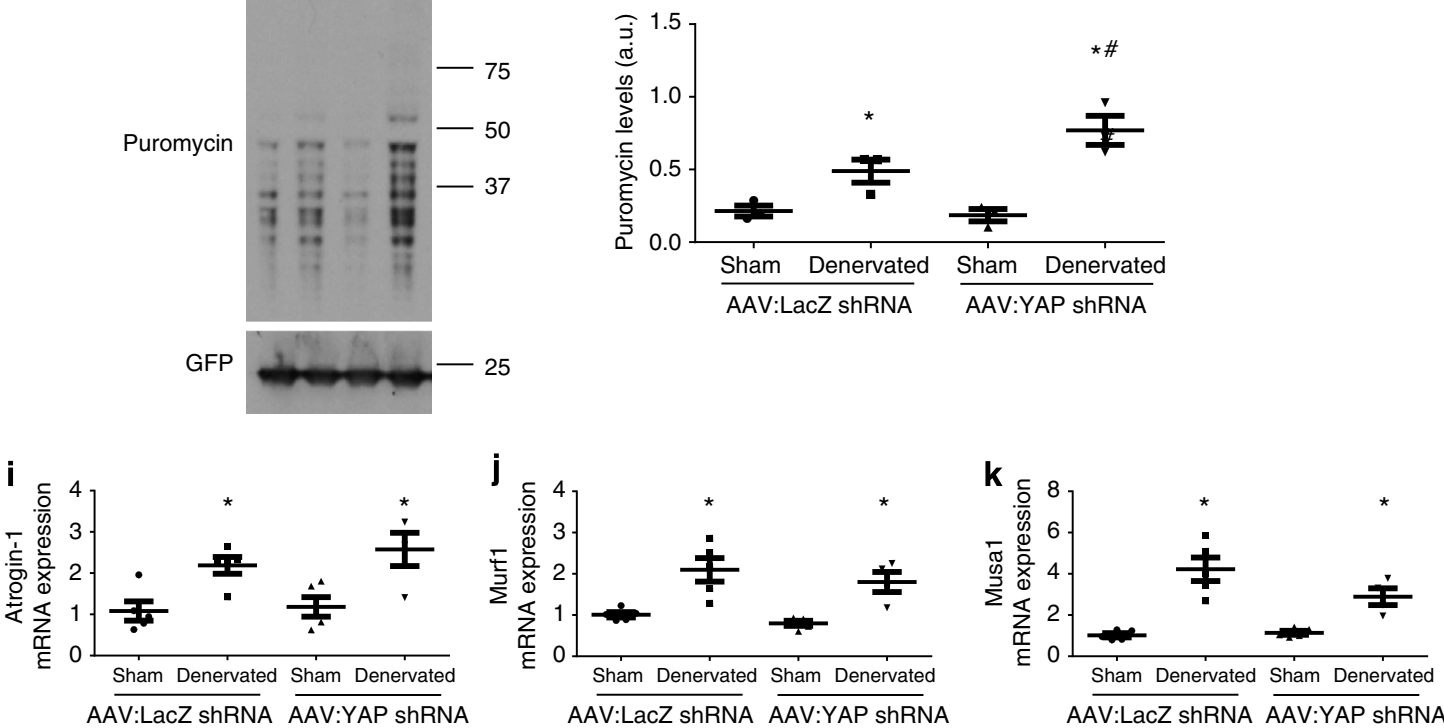
a

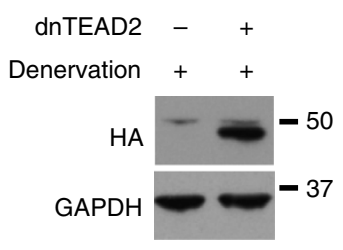

d

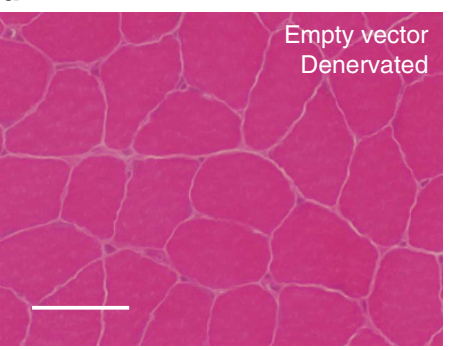

b

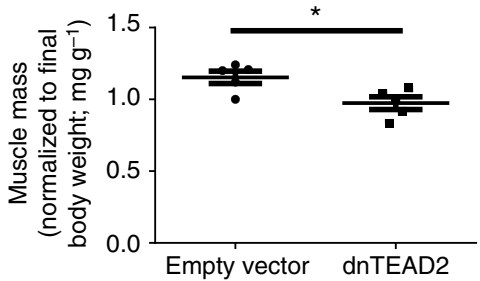

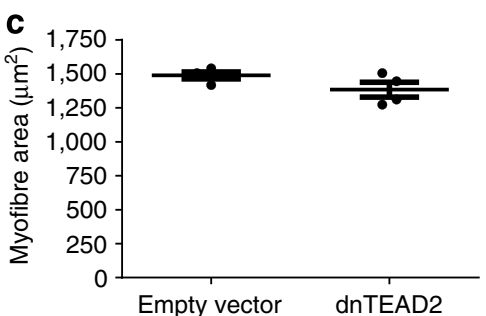

$\mathbf{f}$ e

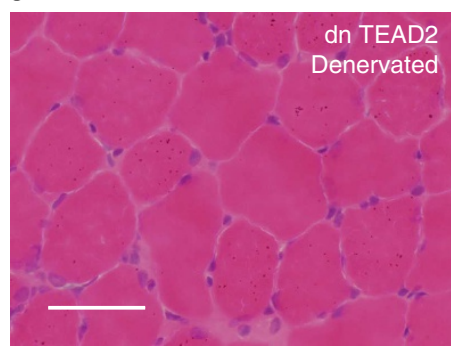

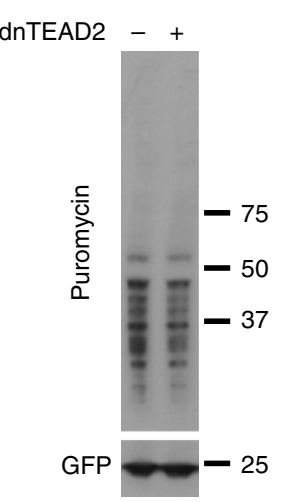

g

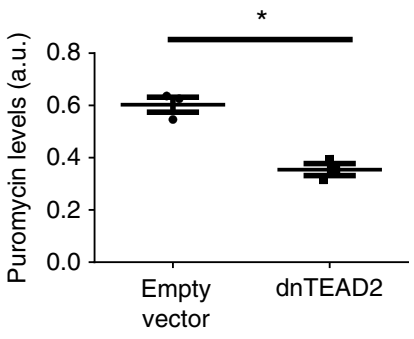

h

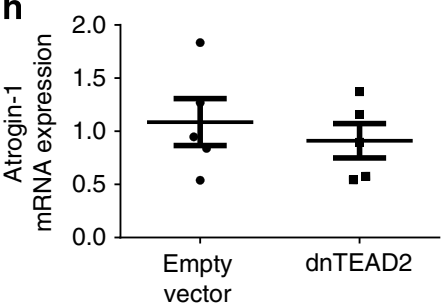

i

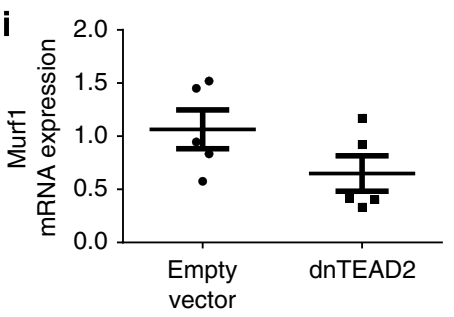

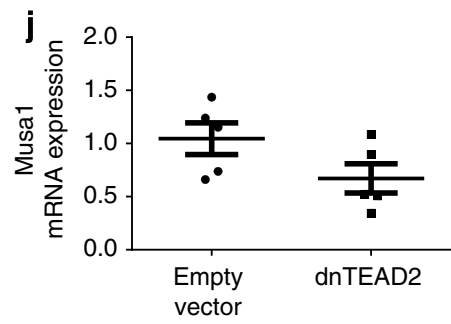

Figure 6 | Inhibition of TEAD transcription factors exacerbates neurogenic muscle atrophy. (a) Representative western blots of HA-TEAD2 and GAPDH in denervated muscles treated with rAAV6:MCS $(-)$ or rAAV6:dnTEAD2 $(+) . n=5$. (b) Muscle mass and (c) myofibre CSA in rAAV6:MCSand rAAV6:dnTEAD2-treated muscles examined 7 days after denervation (mean \pm s.e.m., $n=5, P=0.03$ and 0.18 respectively; paired $t$-test).

(d,e) Representative images of muscle administered rAAV6:MCS and rAAV6:dnTEAD2, then denervated and examined 7 days later. Scale bar, $50 \mu$ m. $n=5$. (f) Puromycin and GFP levels with $(\mathbf{g})$ quantification in rAAV6:MCS $(-)$ and rAAV6:dnTEAD2 $(+)$ treated denervated muscles studied 7 days post denervation (mean \pm s.e.m., $n=3, P=0.02$; paired $t$-test). (h) Atrogin-1, (i) Murf1 and (j) Musa1 mRNA expression in rAAV6:MCS- and rAAV6:dnTEAD2treated denervated muscles (mean \pm s.e.m., $n=5, P=0.6,0.08$ and 0.05 respectively; paired $t$-test).

state of the actin cytoskeleton influences YAP/TAZ localization and activity via F-actin/RhoA signalling, independent of altered $\mathrm{YAP}^{\mathrm{Ser} 112}$ phosphorylation ${ }^{4,5}$. A number of signalling molecules including the dystrophin-glycoprotein complex reside in close proximity to the NMJ at the postsynaptic membrane and operate in conjunction with the cytoskeleton to relay extracellular signals ${ }^{34}$. Given the remodelling of membrane and cytoskeleton architecture that is associated with skeletal muscle growth and atrophy ${ }^{35,36}$, it will be of interest to investigate the potential mechanisms linking mechanical cues, the tensile state of the cytoskeleton and the control of YAP/Hippo signalling in the context of skeletal muscle adaptation and disease.

While the exact mechanisms that control YAP activity in skeletal muscle remain unclear, here we show that changes in myofibre size caused by modulating YAP levels are associated with altered rates of protein synthesis. To date, the regulation of proliferation and differentiation are the only established functions of YAP in cells of the skeletal muscle lineage ${ }^{21,22,37,38}$. Moreover, it has been demonstrated that increasing YAP activity in proliferating, but not quiescent, muscle stem cells (satellite cells) can inhibit their differentiation and support the development of tumours characteristic of embryonic rhabdomyosarcoma ${ }^{37}$.
Although satellite cells can contribute to the growth of adult myofibres, it has been demonstrated extensively that satellite cell recruitment is not required for hypertrophy in adult mammalian skeletal muscle ${ }^{39,40}$. Instead, postnatal muscle mass is maintained by balancing the rates of protein synthesis and degradation ${ }^{24,27}$. While enhanced UPS activity is a key event in muscle atrophy observed in response to loss of motor neuron activity 24 , there is an increasing appreciation of the significance of enhanced protein synthesis in settings of neurogenic atrophy ${ }^{32,41}$. Our demonstration that YAP is induced in the muscle after disruption of the NMJ to promote conservation of mass suggests that a greater understanding of these mechanisms may allow for the identification of novel strategies that could be employed to maintain or increase skeletal muscle mass in settings of neuromuscular disease.

Ectopic expression of YAP has been linked to the progression of a number of human cancers ${ }^{42}$, the capacity for cardiomyocytes to promote regeneration in the heart after myocardial infarction ${ }^{13,19}$ and in the development of Rhabdomyosarcoma ${ }^{37}$. While modulating YAP abundance in a model of neuromuscular disease (SOD1 ${ }^{\mathrm{G} 93 \mathrm{~A}}$ mice) did not preserve mass compared with control muscles, this is likely due to the complexity of the 
mechanisms driving atrophy in this setting ${ }^{43,44}$ and the early symptomatic stages of the disease assessed. Future studies aimed at manipulating YAP activity in this and other neuromuscular disorders are required to understand the therapeutic potential of manipulating the Hippo pathway in skeletal muscle.

In summary, we show that YAP is an essential regulator of myofibre size in adult skeletal muscle via interaction with TEAD transcription factors. Our study also reveals a novel requirement for YAP and TEAD in the maintenance of skeletal muscle mass and myofibre size in conditions of neurogenic atrophy. The identification of the mechanisms that regulate YAP activity may uncover new approaches that could be employed to promote skeletal muscle mass in conditions where the interaction between motor nerve and muscle fibre is compromised.

\section{Materials and methods}

Reagents. Reagents were purchased from Sigma-Aldrich, Australia, unless stated.

Generation of viral vectors. DNA fragments that encode murine YAP2L, YAP2L $^{\text {S112A }}$, YAP2L ${ }^{\text {S79A }}$ and YAP2L lacking both WW domains (YAP2L ${ }^{\text {WW }}$ mutant) were synthesized by Genscript, Piscataway, USA and subcloned into pAAV6-CMV-MCS-SV40pA plasmid. YAP shRNA (5'-CCGGAAGCGCTGAGTT CCGAAATCTCTCGAGTTCGCGACTCAAGGCTTTAGATTTTTC- ${ }^{\prime}$ ) was synthesized within the sequence encoding miR-155 and subcloned into a pAAV6CMV-humanized renilla GFP-SV40pA plasmid. Human TEAD2 was ordered from Applied Biosystems and an HA tag was inserted on the $3^{\prime}$ end by PCR using standard methods. A dominant-negative TEAD2 protein lacking the DNA-binding domain was generated by PCR using the primers $5^{\prime}$-AGGGAAATCCAGTCCAAG-3' (forward) and 5' -CGGGCCCCCGTCACCCC-3' (reverse), then selfligated and subcloned into pAAV6-CMV-MCS-SV40pA plasmid. Protein expression or silencing of endogenous protein was validated in the human embryonic kidney 293 (HEK293) (American Type Culture Collection, USA) cell line. Cells were transfected using Lipofectamine 2000 (Life Technologies, Victoria, Australia) and manufacturer's protocols.

Recombinant adeno-associated viral vectors were generated as described previously ${ }^{45}$. In brief, $10 \mu \mathrm{g}$ of plasmids containing cDNA constructs were co-transfected with $20 \mu \mathrm{g}$ of pDGM6 packaging plasmid into HEK293 cells (seeded $16 \mathrm{~h}$ prior at a density of $3.2-3.8 \times 10^{6}$ cells) using the calcium phosphate precipitate method to generate type- 6 pseduotyped viral vectors. After $72 \mathrm{~h}$, cells and culture medium were collected and homogenized, before clarification, using a $0.22-\mu \mathrm{m}$ filter (EMD Millipore). Vector was purified by affinity chromatography using a HiTrap heparin column (GE Healthcare), ultracentrifuged overnight and resuspended in sterile Ringer's solution. Vector concentration was determined using a customized quantitative PCR reaction (Applied Biosystems).

Ethical approval. All experiments were conducted in accordance with the relevant codes of practice for the care and use of animals for scientific purposes (National Institutes of Health, 1985; and the National Health and Medical Council of Australia, 2004). All animal work performed in this study was conducted with approval from the Alfred Medical Research and Education Precinct Animal Ethics Committee, Melbourne, Australia.

Animal expression studies. C57BL/6 mice of mixed sex were euthanized by cervical dislocation between embryonic day 17 (E17) and 6 weeks of age, and muscles excised for subsequent biochemical and histological analysis. Transgenic SOD1 ${ }^{\text {G93A }}$ mice derived from the B6SJL-TgN (SOD1 ${ }^{\text {G93A) }}$ ) 1 Gur line (The Jackson Laboratory, USA) were backcrossed onto a pure C57BL/6 background. At 60 and 90 days of age, SOD ${ }^{\text {G93A }}$ mice and wild-type littermates were killed by lethal injection (100 $\mathrm{mg} \mathrm{kg}^{-1}$ sodium pentobarbitone, intraperitoneal administration) and muscles were rapidly excised for processing.

Intramuscular injections of rAAV6 vectors. Intramuscular injections of rAAV6 vector were performed in C57BL/6 male mice aged 8 weeks or SOD1 ${ }^{\mathrm{G} 93 \mathrm{~A}}$ female mice aged 60 days. Mice were placed under general anaesthesia $(2 \%$ isofluorane in $\mathrm{O}_{2}$ ) and then administered a subcutaneous injection of carprofen for post-operative analgesia. As a control, the left TA muscles of mice were injected with rAAV6-MCS (empty vector) for overexpression experiments and rAAV6-LacZ shRNA vector for knockdown experiments. Contralateral muscles were injected with rAAV6 vectors expressing murine isoforms of YAP2L, YAP2L ${ }^{\text {S79A }}$, YAP2L $^{\text {S112A, }}$ YAP2L WW mutant, human dnTEAD2 or YAP shRNA as indicated. Viral particles were diluted in $30 \mu \mathrm{l}$ Hank's buffered salt solution to a dosage of $1 \times 10^{9}-1 \times 10^{10}$ vectors genomes as indicated.
Denervation experiments. For surgical denervation of the peroneal nerve, 8 -week-old C57BL/6 male mice were placed under anaesthesia (2\% isofluorane in $\mathrm{O}_{2}$ ) and then given a subcutaneous injection of carprofen for post-operative analgesia. A small incision was made in the skin distal to the knee and the peroneal nerve was exposed. For the expression studies, unilateral denervation was performed where the left peroneal nerve was exposed but not cut (Sham), while the right peroneal nerve was exposed and a small piece $(\sim 1 \mathrm{~mm})$ was excised (denervated). Following closure of the overlying incision and recovery of consciousness, mice were housed for between $6 \mathrm{~h}$ and 14 days post denervation, as indicated. Loss-of-function studies in denervated muscles were performed as above except that both the left and right TA muscles received rAAV6 vectors encoding either rAAV:LacZ shRNA or rAAV:YAP shRNA 14 days before unilateral denervation.

Protein extraction and western blotting. Skeletal muscles were lysed in homogenization buffer (50 mM Tris-HCl, $1 \mathrm{mM}$ EDTA, 1\% (v/v), Triton-X-100, $0.1 \%$ (v/v) 2-mercaptoethanol; $\mathrm{pH} 7.5$ ) supplemented with protease and phosphatase inhibitor cocktail. Protein concentrations were determined using the bicinchoninic acid assay as per the manufacturer's instructions (Thermo Fisher Scientific, Victoria, Australia). Proteins were separated and transferred to nitrocellulose membranes (Bio-Rad Laboratories, Australia) before blocking for $1 \mathrm{~h}$ at room temperature (RT) in 5\% non-fat milk that was diluted in PBS containing $0.1 \%$ Tween-20. Membranes were incubated overnight with primary antibodies followed by incubation for $1 \mathrm{~h}$ at RT with horseradish peroxidase-conjugated secondary antibodies and detection using ECL detection reagent (GE Healthcare Life Sciences, Australia). Primary antibodies used were anti-YAP (\#4912, 1/1,000), anti-pYAP ${ }^{\text {Ser127 }}$ (\#4911, 1/1,000), anti-HA (\#C29F4, 1/1,000), anti-mTOR Ser2448 (\#2971, 1/1,000), anti-mTOR (\#2972, 1/1,000), anti-p70S6K Thr389 $(\# 9205,1 / 1,000)$, p70S6K (\#9202, 1/1,000), p4E-BP1 ${ }^{\operatorname{Ser} 65}$ (\#9451, 1/1,000), 4E-BP1 (\#9452, 1/1,000); all Cell Signalling Technologies, anti-GAPDH (\#SC32233) and anti-CARP/ ANKRD1 (\#SC30181; Santa Cruz Biotechnology, 1/15,000 and 1/100 dilutions, respectively) and anti-Puromycin (\#12D10, Millipore) at a 1/5,000 dilution. Secondary antibodies used were goat anti-rabbit (\#1706516) and goat anti-mouse (\#1706515; Bio-Rad Technologies, both 1/5,000 dilution) except for Puromycin blots where a goat anti-mouse IgG2a conformation specific antibody was used (Abcaus \#115-035-206) at 1/50,000 dilution. Densitometry was performed using ImageJ software (http://rsb.info.nih.gov/ij/index.html) and the levels of GAPDH were used for normalization. Uncropped scans of all western blots shown in Figs 1-6 are provided in Supplementary Figs 12-15.

In vivo estimation of protein synthesis. Protein synthesis was measured using SuNSET $^{25}$. In brief, the mice were injected with $0.04 \mu \mathrm{mol} \mathrm{g}{ }^{-1}$ body weight via an intraperitoneal injection exactly $30 \mathrm{~min}$ before experimental end point. The tissue was processed as described for protein extraction and western blotting. To control for variations in sample loading, cell lysates were supplemented with $3 \mu \mathrm{g}$ of cell lysate from HEK cells transfected with $2 \mu \mathrm{g}$ of pcDNA3.1 plasmid encoding enhanced green fluorescent protein for $48 \mathrm{~h}$. Puromycin levels were then normalized to enhanced green fluorescent protein levels.

Histology and immunofluorescence. Muscles were placed in cryomolds containing optimum cutting temperature (Grale Scientific, Victoria, Australia), frozen in liquid nitrogen-cooled isopentane and then stored at $-80^{\circ} \mathrm{C}$. Haematoxylin and eosin staining was performed as per standard protocols with 10 - $\mu$ m-thick sections and imaged at $\times 400$ magnification on an Olympus FSX-100 microscope. For immunofluorescence labelling, the sections were cut at $5 \mu \mathrm{m}$ and then fixed in $-20^{\circ} \mathrm{C}$ methanol at $-20^{\circ} \mathrm{C}$ for $5 \mathrm{~min}$ at RT, washed twice in KPBS-G (KPBS containing $10 \%$ gelatin) for $5 \mathrm{~min}$ and then blocked in $5 \%$ goat serum in KPBS-G supplemented with $1 \%$ Tween-20 for $30 \mathrm{~min}$ at RT. Sections were incubated in primary antibody diluted in 5\% normal goat serum in KPBS-G overnight at $4{ }^{\circ} \mathrm{C}$. Sections were then incubated in Alex-Fluor-conjugated secondary antibodies and $\alpha$-bungarotoxin that was conjugated to Alex-Fluor 594 (Life Technologies) for $1 \mathrm{~h}$ at RT diluted in KPBS-G, then PBS containing 4',6-diamidino-2-phenylindole at a concentration of $0.5 \mu \mathrm{g} \mathrm{ml}^{-1}$ for $1 \mathrm{~min}$. Sections were washed $3 \times 5 \mathrm{~min}$ in KPBS$\mathrm{G}$ and then mounted in Mowioli. Primary antibodies used for immunofluorescence were anti-YAP (\#4912; Cell Signalling, 1/25) and Laminin (05-206; Merck, 1/250). AchR were labelled using $\alpha$-bungarotoxin conjugated to Alexafluor (AF) 594 at a dilution of $1 / 400$ during the secondary antibody incubation step. Secondary antibodies used were goat anti-rabbit AF488 and goat anti-rat AF647 (Invitrogen, $1 / 200$ dilution). Immunofluorescent labelled sections were imaged as sequential images using a Nikon A1 confocal microscope using a $\times 60$ oil immersion lens. Analysis was performed using ImageJ software. These images were then used to count the numbers of YAP-positive muscle nuclei. A minimum of 50 muscle nuclei were assessed per condition from at least three random fields of view per section.

Assessment of muscle fibre CSA. Sections of muscles were labelled as described for immunofluorescence using rat anti-Laminin antibody (Merck, Australia) overnight at $4{ }^{\circ} \mathrm{C}$. Images were taken using an Olympus BX-61 and analysed using ImageJ software. Myofibre CSA assessments were made from at least 100 muscle fibres taken from three random fields of view per muscle. 
Quantitative real-time reverse transcriptase PCR. Total RNA was extracted from $10-20 \mathrm{mg}$ of skeletal muscle using TRIzol (Life Technologies) as per manufacturer's protocols. Reverse transcription was performed using Multiscribe reverse transcriptase (Life Technologies) as per manufacturer's protocols. Transcripts were measured using Taqman fluorogenic primer probe sets (Life Technologies). Primers used were Yap (Mm01143263_m1), Ankrd1 (Mm00496512_m1), Ctgf (Mm01192932_g1), Atrogin-1 (Mm00499523_m1), Murf1 (Mm01185221_ml) and Musa1 (Mm01191299_m1). The levels of mouse Gapdh (Mm99999915_g1) or 185 (\#4319413E) were used to normalize samples for analysis by the $\Delta \Delta^{C \mathrm{Ct}}$ method.

Statistics. No statistical test was used to predetermine the optimal sample size; however, the sample sizes were similar to those used in previous studies by our group and others. Animals were randomly assigned to experimental groups. Data are presented as mean \pm s.e.m. and are representative of three to six independent biological replicates as indicated in the appropriate figure legends. No animals were excluded from statistical analysis. The investigators were not blinded to allocation during the experiments or to experimental outcome. Two-way analysis of variance (ANOVA) tests were used to assess the statistical differences across multiple conditions with the Bonferroni post hoc test used for comparisons between the specific group means. Comparisons between two conditions used the Student's $t$ test. All differences reported are $P<0.05$.

\section{References}

1. Harvey, K. \& Tapon, N. The Salvador-Warts-Hippo pathway - an emerging tumour-suppressor network. Nat. Rev. Cancer 7, 182-191 (2007).

2. Zhao, B., Tumaneng, K. \& Guan, K. L. The Hippo pathway in organ size control, tissue regeneration and stem cell self-renewal. Nat. Cell Biol. 13, 877-883 (2011).

3. Zhao, B. et al. Inactivation of YAP oncoprotein by the Hippo pathway is involved in cell contact inhibition and tissue growth control. Genes Dev. 21, 2747-2761 (2007).

4. Dupont, S. et al. Role of YAP/TAZ in mechanotransduction. Nature 474, 179-183 (2011).

5. Aragona, M. et al. A mechanical checkpoint controls multicellular growth through YAP/TAZ regulation by actin-processing factors. Cell 154, 1047-1059 (2013).

6. Yu, F. X. et al. Regulation of the Hippo-YAP pathway by G-protein-coupled receptor signaling. Cell 150, 780-791 (2012).

7. Koontz, L. M. et al. The Hippo effector Yorkie controls normal tissue growth by antagonizing scalloped-mediated default repression. Dev. Cell 25, 388-401 (2013).

8. Camargo, F. D. et al. YAP1 increases organ size and expands undifferentiated progenitor cells. Curr. Biol. 17, 2054-2060 (2007).

9. Dong, J. et al. Elucidation of a universal size-control mechanism in Drosophila and mammals. Cell 130, 1120-1133 (2007).

10. Cai, J. et al. The Hippo signaling pathway restricts the oncogenic potential of an intestinal regeneration program. Genes Dev. 24, 2383-2388 (2010).

11. Schlegelmilch, K. et al. Yap1 acts downstream of alpha-catenin to control epidermal proliferation. Cell 144, $782-795$ (2011).

12. Zhang, H., Pasolli, H. A. \& Fuchs, E. Yes-associated protein (YAP) transcriptional coactivator functions in balancing growth and differentiation in skin. Proc. Natl Acad. Sci. USA 108, 2270-2275 (2011).

13. Xin, M. et al. Regulation of insulin-like growth factor signaling by yap governs cardiomyocyte proliferation and embryonic heart size. Sci. Signal. 4, ra70 (2011).

14. von Gise, A. et al. YAP1, the nuclear target of Hippo signaling, stimulates heart growth through cardiomyocyte proliferation but not hypertrophy. Proc. Natl Acad. Sci. USA 109, 2394-2399 (2012).

15. Xin, M. et al. Hippo pathway effector Yap promotes cardiac regeneration. Proc. Natl Acad. Sci. USA 110, 13839-13844 (2013).

16. Heallen, T. et al. Hippo pathway inhibits Wnt signaling to restrain cardiomyocyte proliferation and heart size. Science 332, 458-461 (2011).

17. Heallen, T. et al. Hippo signaling impedes adult heart regeneration. Development 140, 4683-4690 (2013).

18. Matsui, Y. et al. Lats2 is a negative regulator of myocyte size in the heart. Circ. Res. 103, 1309-1318 (2008).

19. Del Re, D. P. et al. Yes-associated protein isoform 1 (Yap1) promotes cardiomyocyte survival and growth to protect against myocardial ischemic injury. J. Biol. Chem. 288, 3977-3988 (2013).

20. Wei, B. et al. MST1, a key player, in enhancing fast skeletal muscle atrophy. BMC Biol. 11, 12 (2013).

21. Watt, K. I. et al. Yap is a novel regulator of $\mathrm{C} 2 \mathrm{C} 12$ myogenesis. Biochem. Biophys. Res. Commun. 393, 619-624 (2010).

22. Judson, R. N. et al. The Hippo pathway member Yap plays a key role in influencing fate decisions in muscle satellite cells. J. Cell Sci. 125, 6009-6019 (2012).

23. Lian, I. et al. The role of YAP transcription coactivator in regulating stem cell self-renewal and differentiation. Genes Dev. 24, 1106-1118 (2010).
24. Schiaffino, S., Dyar, K. A., Ciciliot, S., Blaauw, B. \& Sandri, M. Mechanisms regulating skeletal muscle growth and atrophy. FEBS J. 280, 4294-4314 (2013).

25. Goodman, C. A. et al. Novel insights into the regulation of skeletal muscle protein synthesis as revealed by a new nonradioactive in vivo technique. FASEB J. 25, 1028-1039 (2011).

26. Sartori, R. et al. BMP signaling controls muscle mass. Nat. Genet. 45 , 1309-1318 (2013).

27. Bonaldo, P. \& Sandri, M. Cellular and molecular mechanisms of muscle atrophy. Dis. Model Mech. 6, 25-39 (2013).

28. Bodine, S. C. et al. Identification of ubiquitin ligases required for skeletal muscle atrophy. Science 294, 1704-1708 (2001).

29. Komuro, A., Nagai, M., Navin, N. E. \& Sudol, M. WW domain-containing protein YAP associates with ErbB-4 and acts as a co-transcriptional activator for the carboxyl-terminal fragment of ErbB-4 that translocates to the nucleus. J. Biol. Chem. 278, 33334-33341 (2003).

30. Zhao, B. et al. TEAD mediates YAP-dependent gene induction and growth control. Genes Dev. 22, 1962-1971 (2008).

31. Judson, R. N. et al. Constitutive expression of Yes-associated protein (Yap) in adult skeletal muscle fibres induces muscle atrophy and myopathy. PLOS ONE 8, e59622 (2013).

32. Quy, P. N., Kuma, A., Pierre, P. \& Mizushima, N. Proteasome-dependent activation of mammalian target of rapamycin complex 1 (mTORC1) is essential for autophagy suppression and muscle remodeling following denervation. J. Biol. Chem. 288, 1125-1134 (2013).

33. Yu, F. X. et al. Protein kinase A activates the Hippo pathway to modulate cell proliferation and differentiation. Genes Dev. 27, 1223-1232 (2012).

34. Shi, L., Fu, A. K. \& Ip, N. Y. Molecular mechanisms underlying maturation and maintenance of the vertebrate neuromuscular junction. Trends Neurosci. 35, 441-453 (2012).

35. Hornberger, T. A. Mechanotransduction and the regulation of mTORC1 signaling in skeletal muscle. Int. J. Biochem. Cell. Biol. 43, 1267-1276 (2011).

36. Burkholder, T. J. Mechanotransduction in skeletal muscle. Front. Biosci. 12, 174-191 (2007)

37. Tremblay, A. M. et al. The Hippo transducer YAP1 transforms activated satellite cells and is a potent effector of embryonal rhabdomyosarcoma formation. Cancer Cell 26, 273-287 (2014).

38. Gee, S. T., Milgram, S. L., Kramer, K. L., Conlon, F. L. \& Moody, S. A. Yes-associated protein 65 (YAP) expands neural progenitors and regulates Pax3 expression in the neural plate border zone. PLOS ONE 6, e20309 (2011).

39. Jackson, J. R. et al. Satellite cell depletion does not inhibit adult skeletal muscle regrowth following unloading-induced atrophy. Am. J. Physiol. Cell Physiol. 303, C854-C861 (2012).

40. McCarthy, J. J. et al. Effective fiber hypertrophy in satellite cell-depleted skeletal muscle. Development 138, 3657-3666 (2011).

41. Gordon, B. S., Kelleher, A. R. \& Kimball, S. R. Regulation of muscle protein synthesis and the effects of catabolic states. Int. J. Biochem. Cell Biol. 45, 2147-2157 (2013).

42. Harvey, K. F., Zhang, X. \& Thomas, D. M. The Hippo pathway and human cancer. Nat. Rev. Cancer 13, 246-257 (2013).

43. Dobrowolny, G. et al. Skeletal muscle is a primary target of SOD1G93Amediated toxicity. Cell Metab. 8, 425-436 (2008).

44. Dupuis, L. \& Echaniz-Laguna, A. Skeletal muscle in motor neuron diseases: therapeutic target and delivery route for potential treatments. Curr. Drug Targets 11, 1250-1261 (2010).

45. Winbanks, C. E. et al. The bone morphogenetic protein axis is a positive regulator of skeletal muscle mass. J. Cell Biol. 203, 345-357 (2013).

\section{Acknowledgements}

We thank Dr Rachel Thomson for technical assistance and Dr Iska Carmichael, Monash Micro Imaging facility, AMREP campus, BakerIDI. This work was supported by Project Grant funding (1009995 awarded to P.G. and K.F.H., 1008910 awarded to B.J.T.) from the Australian National Health and Medical Research Council (NHMRC). K.F.H. is a Sylvia and Charles Viertel Senior Medical Research Fellow. P.G. is supported by a Career Development Fellowship (1046782) from the NHMRC, and was previously supported by a Senior Research Fellowship, sponsored by Pfizer Australia. B.J.T. is supported by the Susie Harris Memorial Fund, MND research grant, MND Research Institute of Australia The Baker IDI Heart and Diabetes Institute and The Florey Institute of Neuroscience and Medical health are supported in part by the Operational Infrastructure Support Program of the Victorian Government.

\section{Author contributions}

K.I.W., K.F.H. and P.G. designed the studies. K.I.W., B.J.T., A.H., X.Z., J.R.D., H.Q., C.B C.E.W. and P.G. performed the experimental work. K.I.W., B.J.T., A.H., K.F.H. and P.G. analysed the results. K.I.W., K.F.H. and P.G. wrote the manuscript. 


\section{Additional information}

Supplementary Information accompanies this paper at http://www.nature.com/ naturecommunications

Competing financial interests: The authors declare no competing financial interests.
Reprints and permission information is available online at http://npg.nature.com/ reprintsandpermissions/

How to cite this article: Watt, K. I. et al. The Hippo pathway effector YAP is a critical regulator of skeletal muscle fibre size. Nat. Commun. 6:6048 doi: 10.1038/ncomms7048 (2015). 